\title{
A NEW SELF-SIMILAR TRAFFIC MODEL AND ITS APPLICATIONS IN TELECOMMUNICATION NETWORKS
}

\author{
David G. Daut ${ }^{1}$ and Ming $\mathrm{Yu}^{2}$ \\ ${ }^{1}$ Rutgers - The State University \\ ${ }^{2}$ AT\& T Labs \\ of New Jersey \\ 480 Red Hill Road \\ Dept. of Elec. \& Comp. Eng. \\ Room $1 D-328$ \\ Piscataway, NJ 08855 \\ Middletown, NJ 07748 \\ USA \\ USA \\ daut@ece.rutgers.edu \\ mingyu@att.com
}

\begin{abstract}
This paper presents a new self-similar traffic model derived from the arrival processes of $M / G / \infty$ queue. It has a structure similar to that of a fractional ARIMA, with a driven process of $\mathrm{fBm}$ (fractional Brownian motion). The coefficients of the $\mathrm{fBm}$ are derived from the Pareto distribution of the active periods of the arrival process. When applied to a single server with self-similar input, the model results in an explicit buffer level equation which matches Norros' storage model. So this method can be also served as a verification of Norros' assumptions. The effectiveness of the proposed model has been verified by some practical examples.
\end{abstract}

Keywords: Self-similar, long-range dependence, network traffic.

\section{INTRODUCTION}

Network traffic modeling is of primary importance for network design, performance prediction, and control. It has been observed recently that packet loss and delay are more serious than expected because network traffic is more bursty and exhibits greater variability than previously suspected. This phenomenon has led to the discovery of network traffic's self-similar, or fractal, characteristic [6]. A covariance-stationary process $X(t)$ is called self-similar if $X(t)-X(0)$ and $r^{H}(X(u)-X(0))$ are identical in distribution, where the time $t$ is rescaled in the ratio $r$, i.e., $u=t / r$. Therefore the area of network traffic modeling, buffer design, and performance evaluation needs to be reexamined. 
There are many investigations into self-similar models. The fractional Brownian motion (fBm) and fractional Auto-Regressive Integrated Moving Average (f-ARIMA) model are the two popular mathematical models that are used to describe self-similar processes. F-ARIMA is much more flexible than $\mathrm{fBm}$. But both can be only used to simulate short time series. Cox and Isham proposed a model to describe an immigration-death process. Kosten applied this model to a multi-entry buffer in the general context of self-similarity. There are two other related models of asymptotically self-similar processes. One is a sum of short-range independent attenuated and weighted stationary processes. Another is taking the limit of the aggregated traffic of $M$ individual On-Off sources, as $M \longrightarrow \infty$, while keeping the source rate and the distribution of active period remain unchanged, but the distribution of idle period went to zero. The $M / G / \infty$ and aggregated $A R(1)$ processes are the two models often used to generating asymptotically self-similar traffic although without analytical expressions. Other self-similar models are heavy tailed On-Off process, stable self-similar process, fractal shot-noise (point process approaches), fractal renewal process (point process approaches) [3], deterministic nonlinear chaotic map models, and stochastic difference equations.

The complexity of self-similarity has rendered existing traffic and performance models to be either analytically intractable in the evaluation of performance, or usually inaccurate with respect to dynamic queueing behavior. In order to investigate the impact of self-similar traffic modeling parameters on network performance, it is necessary to develop new analytic traffic models.

\section{CONSTRUCTION OF SELF-SIMILAR TRAFFIC}

Consider a stationary random process $Y=\left\{Y_{t}\right\}$, where $t \in I_{-\infty}=$ $\{\ldots,-1,0,1, \ldots\}$. We assume that there are $\xi_{t}$ traffic sources which begin their active periods at time $t$, where $\xi_{t}$ is independent and Poisson with mean $\lambda_{1}$. A source $s$ is associated with its active period $s$, where $s \in I_{-\infty}$. The beginning instant of active period $s$ is denoted by $\omega_{s}$. The length of active period $s$ is denoted by $\tau_{s} \in I_{1}=\{1,2, \ldots\}$. The number of cells generated by source $s$ at time instant $t$ is denoted by $\vartheta_{t}^{(s)}$, which is also called the source rate of source $s$. In this section, we will focus on the derivation of our self-similar traffic model instead of the procedure of traffic construction. Details can be found in [7]. The number of new cells $Y_{t}$ that appeared in traffic $Y$ at time $t$ is the sum of numbers of cells generated by all active (at time $t$ ) sources, including those sources began at time $t$ and those began before time $t$ but still in active at time $t$,

$$
Y_{t}=\sum_{s=-\infty}^{\infty} \vartheta_{t}^{(s)}
$$


If the source rate is constant, $\hat{\vartheta}_{t}^{(s)}=R \in I_{1}$, the distribution of $Y_{t}$ for given $t$ is Poisson. If the source rate is Poisson, $\hat{\vartheta}_{t}^{(s)}$ are independent (for all $s$ and $t$ ) and Poisson with mean $\lambda_{2}$. The intensity of this traffic is $E\left\{Y_{t}\right\}=\lambda_{1} \lambda_{2} E\{\tau\}$. However, the distribution of $Y_{t}$ is not Poisson (since the sum of a random number of Poisson random variables is not necessarily a Poisson variable). Moreover, $Y_{t}$ are dependent variables. We will find a mathematical model for this process for the purpose of queueing analysis and prove its self-similarity.

The number of new cells $Y_{t}$ at time $t$ can be expressed by

$$
Y_{t}=\sum_{n=-\infty}^{t} R \xi_{n} P_{r o b}^{(n)}(\tau>t-n)
$$

where $P_{r o b}^{(n)}(\tau>l)$ is the distribution of the length of active period at time $n$. If $P_{r o b}^{(n)}=P_{r o b}$, for all $n$; let $t-n=l$, then we have

$$
Y_{t}=\sum_{l=0}^{\infty} R \xi_{t-l} P_{r o b}(\tau>l)
$$

The above model can be rewritten as

$$
Y_{t}=R P_{\text {rob }}(\tau>0) \xi_{t}+R P_{\text {rob }}(\tau>1) \xi_{t-1}+R P_{\text {rob }}(\tau>2) \xi_{t-2}+\ldots
$$

After some rearrangement, we have

$$
\frac{1}{R} Y_{t+1}-\frac{1}{R} Y_{t}=\xi_{t+1}-a_{1} \xi_{t}-a_{2} \xi_{t-1}-\ldots-a_{-\infty} \xi_{-\infty}
$$

where $a_{j}=P_{\text {rob }}(\tau>j-1)-P_{\text {rob }}(\tau>j)=P_{\text {rob }}(\tau=j), j=1,2,3, \ldots$, $\left\{\xi_{j}\right\}$ is Poisson. Suppose the distribution $P_{\text {rob }}(\tau \geq l+1)$ is Pareto-type,

$$
P_{\text {rob }}(\tau \geq l+1)=\frac{E \tau}{2} \beta(1-\beta)(2-\beta) l^{-(\beta+1)}, \beta \rightarrow \infty .
$$

the process $Y_{t}$ will be proven to be self-similar with $H=1-\frac{\beta}{2}, 0<\beta<1$ (see next section).

A Poisson process $N(t)$ with parameter $m$ can be approximated by a diffusion process

$$
N(t) \approx m t+\sqrt{a m} W(t)
$$

Substitute $\xi$ in Eqn. (5) for $N(t)$ in Eqn. (7), and note that $\sum_{j=0}^{\infty} a_{t-j}=1$, and $\sum_{j=1}^{\infty} a_{t-j} j=E \tau$, we have

$$
Y_{t+1}-Y_{t}=m R E \tau+R \sqrt{a m} z(t+1),
$$


where $m$ is the diffusion approximation parameter; $a$ is the ratio of the variance to the mean of the traffic process; $\tau$ is the length of an active period; $z(t)$ is a fractional Brownian motion which can be expressed as

$$
z(t+1)=W(t+1)-a_{t} W(t)-a_{t-1} W(t-1)-\ldots-a_{-\infty} W(-\infty),
$$

where the coefficients $a_{t}$ are the probabilities of the lengths of the active periods; and $W(t)$ is the standard Brownian motion.

\section{SELF-SIMILARITY OF THE NEW MODEL}

In this section, we attempt to give a rough proof of the self-similarity of the traffic process $Y_{t}$ represented by the new model developed in the previous section. Before we present our proof, we need to introduce an LRD property:

A covariance-stationary process $X=(X ; i=1,2,3, \ldots)$ with mean $\mu$, variance $\sigma^{2}$ and autocorrelation function $r(k), k \geq 0$, is self-similar iff $X$ is long-range dependence (LRD), i.e., for some $\frac{1}{2}<r<1$,

$$
r(k) \sim c k^{2 H-2} \text { as } k \rightarrow \infty
$$

where $c$ is a finite positive constant. This property can be derived from the definition fo LRD and its relationship to self-similarity.

Define the auto-covariance function of $Y_{t}$,

$$
\omega(k)=\operatorname{cov}\left\{Y_{t}, Y_{t+k}\right\}=E\left\{\left(Y_{t}-E\left\{Y_{t}\right\}\right)\left(Y_{t+k}-E\left\{Y_{t+k}\right\}\right)\right\}
$$

because of its stationary, we have $\omega(k)=r(k)-\left(E\left\{Y_{t}\right\}\right)^{2}$. Therefore $\omega(k)$ and $r(k)$ will have the same asymptotic property for large $k$. Now the problem becomes to prove that $\omega(k)$ will have the form of Eqn. (10) for large $k$. What we know is that $Y_{t}$ has the form of Eqn. (8) and $z(t)$ is $\mathrm{fBm}$ which can be expressed by $W(t)$, standard Bm.

From $Y_{t+1}=Y_{t}+m R \bar{\tau}+R \sqrt{a m} z(t+1)$, we have

$$
\begin{gathered}
Y_{t+k}=Y_{t}+k m R \bar{\tau}+R \sqrt{a m}[z(t+1)+z(t+2)+\ldots+z(t+k)] \\
Y_{t+k}-E Y_{t+k}=Y_{t}-E Y_{t}+\sqrt{a m} R[z(t+1)+z(t+2)+\ldots \\
+z(t+k)-\bar{z}(t+1)-\bar{z}(t+2)-\ldots-\bar{z}(t+k)] \\
\omega(k)=E\left\{\left(Y_{t}-\bar{Y}_{t}\right)^{2}\right\}+m a R^{2}\left[\omega_{z}(0)+\omega_{z}(1)+\ldots+\omega_{z}(k)\right]
\end{gathered}
$$

where $\omega_{z}(k)=E\{[z(t)-\bar{z}(t)][z(t+k)-\bar{z}(t+k)]\}$. Note that $\bar{z}(t+i)=$ $0, i=0,1,2, \ldots, k$. We have 


$$
\begin{gathered}
z(t+i)=W(t+i)-a_{1} W(t+i-1)-a_{2} W(t+i-2)-a_{3} W(t+i-3)-\ldots \\
-a_{i-1} W(t+1)-a_{i} W(t)-a_{i+1} W(t+1)-\ldots-a_{-\infty} W(-\infty),
\end{gathered}
$$

Also note $W(i) W(j)=\delta_{i j} \sigma^{2}$ Suppose $\sigma^{2}=1$, we have

$$
\omega_{z}(i-1)=E\{z(t+1) z(t+i)\}=1 a_{i-1}+a_{1} a_{i}+a_{2} a_{i+1}+a_{3} a_{i+2}+\ldots
$$

Because of the Pareto-type distribution of the active periods: $a_{i}=c i^{-\alpha-1}$, where $\mathrm{c}$ is constant, which can be determined from $\bar{\tau}$ and $\beta$. Thus

$$
\omega_{z}(i)=c i^{-\alpha-1}+\sum_{n=1}^{\infty} c^{2} n^{-\alpha-1}(i+n)^{-\alpha-1},
$$

We know $E\left\{\left(Y_{t}-E Y_{t}\right)^{2}\right\}=R^{2}(E \xi)(E \tau)=R^{2} m \bar{\tau}$ therefore

$$
\omega(k)=m R^{2} \bar{\tau}+a m R^{2} \sum_{j=0}^{k} \omega_{z}(j)
$$

when $n_{0}$ is large, using

$$
\sum_{n=n_{0}}^{\infty} n^{-\alpha} \approx \frac{n_{0}^{-\alpha+1}}{\alpha-1}
$$

therefore when $k$ is large we have

$$
\begin{gathered}
\sum_{n=1}^{\infty}\left(n k+n^{2}\right)^{-(\alpha+1)} \approx \frac{(k+1)^{-\alpha}}{\alpha}, \\
\sum_{j=0}^{k} \omega_{z}(j) \approx \sum_{j=0}^{k}\left[c j^{-\alpha-1}+c^{2} \frac{(j+1)^{-\alpha}}{\alpha}\right] \\
\sum_{j=0}^{k} \omega_{z}(j) \approx c \frac{k^{-\alpha}}{\alpha}+\frac{c^{2}}{\alpha} \frac{(k+1)^{-\alpha+1}}{\alpha-1} \\
\omega(k) \approx \frac{c^{2}}{\alpha(\alpha-1)}(k+1)^{1-\alpha}
\end{gathered}
$$

That is, $\omega(k)$ has the form of Eqn. (10). Therefore $Y_{t}$ is a self-similar process, with Hurst parameter $H=(3-\alpha) / 2$ according to the LRD property. So far, we've developed a new self-similar traffic model and proven that the traffic sequences of $\left\{Y_{t}\right\}$ is self-similar with parameter $H=(3-\alpha) / 2$, which will be used to analytically investigate the queueing behavior of networks with self-similar traffic input. 


\section{QUEUEING ANALYSIS \\ 4.1 BUFFER LEVEL EQUATION}

While there are several excellent papers dedicated to the queueing analysis for self-similar traffic, there is still a lack of exact results concerning queueing analysis such as queue length distributions. Interested readers may check the references $[4 ; 1 ; 2 ; 5]$. Norros' storage model has been widely used to estimate asymptotic performance bounds in the case of large buffers, although it has not been proven because of the complexity of the problem. In this Section, we will apply the new traffic model to develop a buffer level equation that can be used to determine the multiplexing behavior of self-similar traffic and the bounds of the queue length. This development can be also served as a proof of Norros' model.

The new self-similar traffic model can be expanded as

$$
Y_{t}=R P_{r o b}(\tau>0) \xi_{t}+R P_{r o b}(\tau>1) \xi_{t-1}+R P_{r o b}(\tau>2) \xi_{t-2}+\ldots
$$

Define the coefficients using the notation

$$
b_{j}=P_{r o b}(\tau>j)
$$

then, we have

$$
Y_{t}=R b_{0} \xi_{t}+R b_{1} \xi_{t-1}+R b_{2} \xi_{t-2}+\ldots
$$

Similar to Section 2, the Poisson process $\xi(t)$ with parameter $m$ can be written in the form

$$
\xi(t)=m t+W(t)
$$

where $W(t)$ is the martingale $\xi(t)-m t$. It is well known that $\xi(\alpha t)-$ $\alpha m t) / \sqrt{\alpha m}$ converges towards the standard Brownian motion $W(t)$ as $\alpha \rightarrow$ $\infty$. This suggests the approximation of $\xi(t)$ by a diffusion process

$$
\xi(t) \approx m t+\sqrt{a m} W(t)
$$

and upon substitution into our model, we have

$$
Y_{t}=R \sum_{j=0}^{\infty} b_{j} m t-R m \sum_{j=0}^{\infty} j b_{j}+R \sqrt{a m} \sum_{j=0}^{\infty} b_{j} W(t-j) .
$$

Note that

$$
\sum_{j=0}^{\infty} b_{j}=\bar{\tau}
$$


and also

$$
\sum_{j=0}^{\infty} j b_{j}=D(\bar{\tau}, \beta)
$$

is a constant which is related the distributions of the length of active periods. Therefore the model can be simplified as

$$
Y(t)=m t-D(\bar{\tau}, \beta) / \bar{\tau}+\sqrt{a m} Z(t)
$$

where $Y(t)=Y_{t} / R \bar{\tau}$ being defined as the normalized traffic process; $Z(t)$ being defined as

$$
Z(t)=\sum_{j=0}^{\infty} \frac{b_{j}}{\bar{\tau}} W(t-j)
$$

is a normalized version of the fractional Brownian motion $z(t)$ in the new model. Suppose the system has a constant leak rate $C$, the net input process is

$$
X(t)=Y(t)-C t .
$$

Thus, the stationary storage model with fractional Brownian net input is the stochastic process $V(t)$, where

$$
V(t)=\sup _{s \leq t}(Y(t)-Y(s)-C(t-s)), t \in(-\infty, \infty),
$$

That is equivalent to the process

$$
V(t)=\sup _{s \leq t}(A(t)-A(s)-C(t-s)), t \in(-\infty, \infty),
$$

with an arrival process of

$$
A(t)=m t+\sqrt{a m} Z(t)
$$

It is similar to the well-known expression for the amount of work (or virtual waiting time) in a queueing system with service rate $C$ and cumulative work arrival process $A(t)$ [4].

\subsection{PERFORMANCE EVALUATIONS}

The performance requirement in telecommunication applications can be described by following relation

$$
\epsilon=P_{r o b}(V>x)
$$

that the probability that the queue length exceeds a certain level $x$ is required to be at most equal to a "Quality of Service" parameter $\epsilon$. Norros' lower 
bound is asymptotically (in a logarithmic sense) exact for the Brownian model. Massoulie and Simonian have reported a tighten lower and upper bounds which have only a constant underdetermined. Recently, Narayan found an exact asymptotic queue lenght distribution for $1 / 2<H<1$. For case of $H=1$, Norros' lower bound is the exact probability. In this paper we will apply these bounds to a practical example to verify our models.

\section{EXAMPLES}

Example 1 A set of practical traffic data we collected from a large-scale data network was plotted in Fig.1. The Pox diagram of the traffic data is shown in Fig.2. Fig. 3 is the power spectrum.

The traffic $Y_{t}$ can be modeled by

$$
Y_{t+1}-Y_{t}=m R E\{\tau\}+R \sqrt{a m} z(t+1),
$$

where $m=0.5709$ is a diffusion approximation parameter; $a=1.4194$; $\tau=1.9977$ is the length of an active period; the source rate $R=1.0 ; z(t)$ is a fractional Brownian motion which can be expressed as

$$
z(t+1)=W(t+1)-a_{t} W(t)-a_{t-1} W(t-1)-\ldots-a_{-\infty} W(-\infty),
$$

where the coefficients $a_{t}=c t^{\alpha-1}, c=0.7188, \alpha=3-2 H=1.3800, H=$ 0.81 ; and $W(t)$ is the standard Brownian motion. This model has been successfully used to design an alarm processor in a larg-scale network. Fig.4 is the Pox diagram of the traffic generated by this model.

Example 2 The performance bound has been calculated from the proposed buffer level equation when a single sever fed by the above traffic data. In order to compare the bound with other performance bounds, it is plotted in Fig.5 (lower line) against the bound derived from Reference [2]. The traffic would be modeled as Poisson arrival process if self-similarity was ignored. The resulted performance bound was shown in Fig. 6 also against the bound derived from Reference [2]. It can be seen that the performance will be much worse than predicted by Poisson models. This has also demonstrated the need of self-similar traffic model.

\section{CONCLUSION}

In this paper, we developed a new tractable model for self-similar traffic processes. We also derived an explicit buffer level equation based on the proposed traffic model, which matches Norros' storage model. So this method can be also served as a verification of Norros' assumptions. The queueing behavior of a single server to self-similar input can be analytically investigated with the proposed model with respect to each of the model parameters. This 


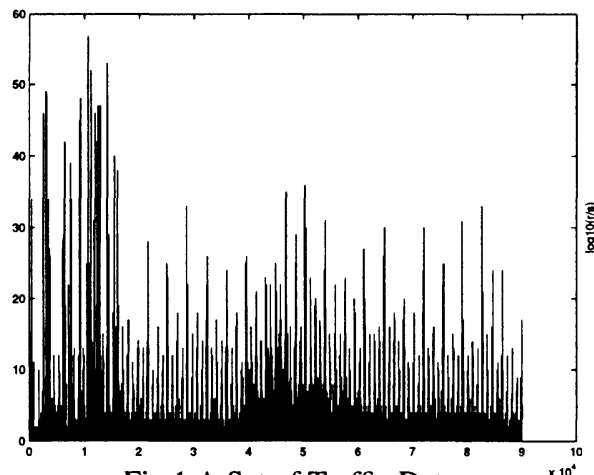

Fig.1 A Set of Traffic Data

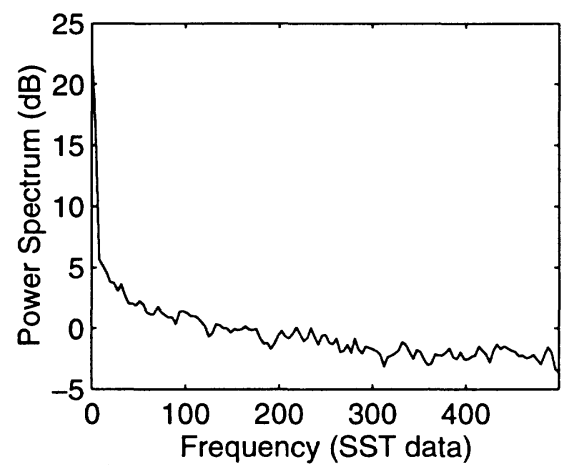

Fig. 3 Power Spectrum of the Traffic

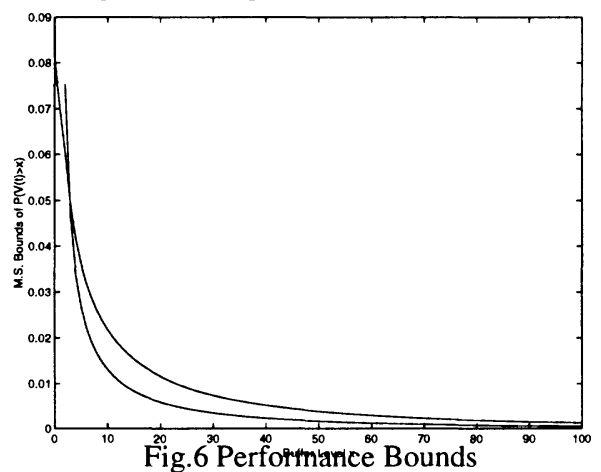

Pox Diagram al Alarm Trastic

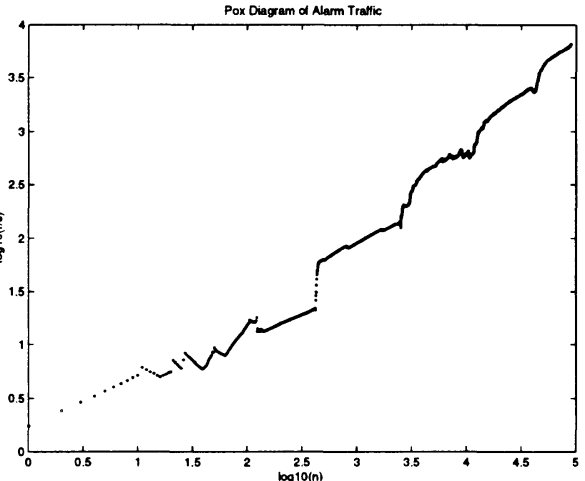

Fig.2 Pox Diagram of the Traffic

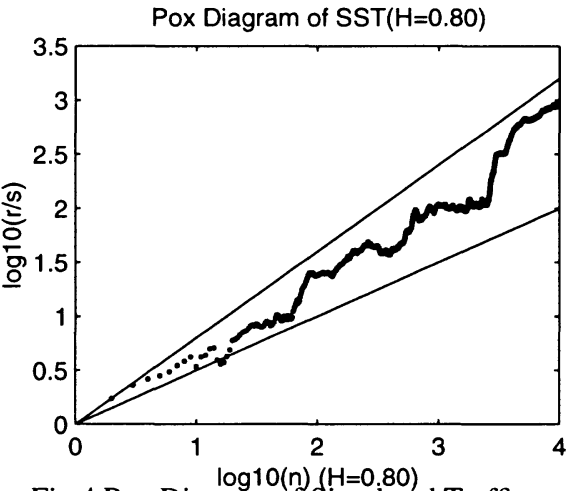

Fig.4 Pox Diagram of Simulated Traffic

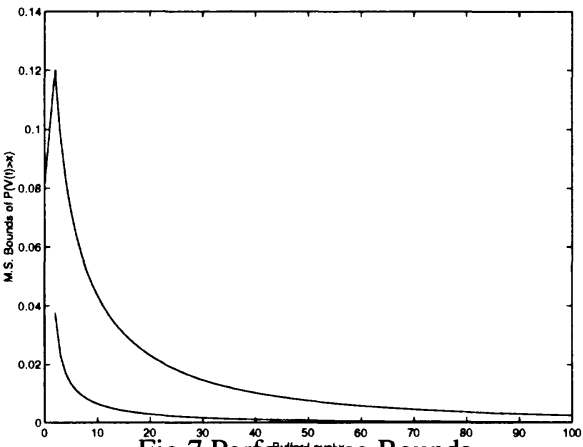

Fig. $7^{30}$ Performance Bounds

will be our future work. Finally, we presented some practical examples to demonstrate the effectiveness of the proposed methods. 


\section{Acknowledgments}

The authors would like to acknowledge the reviewers for their many suggestions and comments. We also thank N.G.Duffield, W.Willinger, and V. Ramaswami for giving us very useful references.

\section{References}

[1] O. Narayan, "Exact Asymptotic Queue Length Distribution for Fractional Brownian Traffic,", Advance in Performance Analysis, vol.1, pp. 39-63, no. 1, Oct. 1998.

[2] L. Massoulie and A. Simonian, "Large Buffer Asymptotics for the Queue with FBM Input,", unpulished, 1998.

[3] W. E. Leland, M. S. Taqqu, W. Willinger, , and D. V. Wilson. On the selfsimilar nature of ethernet traffic. IEEE/ACM Transactions on Networking, vol. 2:1-14, March 1994.

[4] I. Norros. A storage model with self-similar input. Queueing Systems, vol. 16:387-396, 1994.

[5] N. G. Duffield. Large deviatioins and overflow probabilities for the general single-server queue, with applications. Math. Proc. Camb. Phil. Soc., vol. 118:363-374, 1995.

[6] W. Stallings. Self-similarity upsets data traffic assumptions. IEEE Spectrum, vol. 34:28-29, 1997.

[7] B. Tsybakov and N. D. Georganas. Overflow probability in an atm queue with self-similar traffic. In ICC'97, 1997. 